Abstract 63 Table 1 Characteristics of patients with and without MetS and relationship MetS and SLE with NLR

\begin{tabular}{|c|c|c|c|}
\hline & MetS $n=13$ & Non MetS $n=17$ & \\
\hline $\begin{array}{l}\text { Female }(\%) \\
\text { Age years }( \pm \text { SD) } \\
\text { Smoking exposure }(\%) \\
\text { NLR (IQR) } \\
\text { BMI ( } \pm \text { SD) } \\
\text { Nephritis }(\%) \\
\text { Proteinuria gr/24 hrs ( } \pm \text { SD) } \\
\text { Class III / IV } \\
\text { CRP mg/dl ( } \pm \text { SD) } \\
\text { aPL }+(\%) \\
\text { SLEDAI }( \pm \text { SD) } \\
\text { SLICC }( \pm \text { SD) } \\
\text { Accumulated corticoid gr ( } \pm \text { SD) } \\
\text { HCQ }(\%) \\
\text { ASA }(\%)\end{array}$ & $\begin{array}{l}10(77) \\
38.6(12.7) \\
5(38) \\
3.42(1.28-5.67) \\
31(7.2) \\
7(54) \\
1.5(3.19) \\
4 / 7(57) \\
8.7(10) \\
6(46) \\
2.8(3.5) \\
0.23(0.43) \\
6.74(4.6) \\
12(92) \\
3(23)\end{array}$ & $\begin{array}{l}13(76) \\
40(15.7) \\
6(35) \\
3.05(1-6) \\
25(4) \\
10(58) \\
0.35(0.54) \\
6 / 10(60) \\
7.61(22) \\
4(23) \\
2.4(2.52) \\
0.23(0.43) \\
9.43(5.9) \\
17(100) \\
4(24)\end{array}$ & $\begin{array}{l}0.49 \\
0.62 \\
0.43 \\
0.52 \\
0.02 \\
0.39 \\
0.03 \\
0.45 \\
0.02 \\
0.11 \\
0.74 \\
0.97 \\
0.25 \\
0.21 \\
0.49\end{array}$ \\
\hline & NLR ( $\pm \mathrm{DS})$ & $p$ & \\
\hline $\begin{array}{l}\text { MetS } \\
\text { MetS } \geq 4 \text { criteria } \\
\text { Nephritis } \\
\text { SLEDAI } \geq 4 \\
\text { CRP> } 5\end{array}$ & $\begin{array}{l}3.42(1.32) \\
3.77(3.77) \\
3.35(1.62) \\
4.43(1.42) \\
3.87(1.50)\end{array}$ & $\begin{array}{l}0.52 \\
0.61 \\
0.67 \\
0.027 \\
0.11\end{array}$ & \\
\hline
\end{tabular}

\section{ANA BY INDIRECT IMMUNOFLUORESCENCE ALONE RARE IN SLE AND CLINICAL PHENOTYPE OF PATIENTS WITH ANA PLUS LUPUS ASSOCIATED ANTIBODIES IS DIFFERENT THAN ANA ALONE}

H Michael Belmont*, Peter Izmirly, Jill Buyonm. NYU School of Medicine

\subsection{6/lupus-2019-Ism.64}

Background Autoantibody and laboratory testing is essential for SLE diagnosis. ANA by indirect immunofluorescence (ANA IIF) remains the gold standard to screen for lupus and studies demonstrate preclinical phase during which autoantibodies accumulate. Prevalence of ANA IIF alone without more specific autoantibodies and the accompanying clinical phenotype of these patients uncertain.

Methods Queried 602 patients in the NYU Lupus Registry with 4 or more ACR or SLICC criteria as adjudicated by the authors for prevalence of ANA IIF, dsDNA, Sm, Ro, La, RNP, aPL, C3, C4. Compared clinical features of isolated ANA (ANA IIF alone) with the ANA IIF plus one or more lupus associated abnormalities (ANA IIF +).

Results 590/602 (98\%) ANA IIF positive. 548/590 (93\%) patients at least one of associated tests compared to only 42/ 590 (7\%) ANA IIF alone. SLE nephritis significantly more prevalent in ANA IIF+254/548 (46\%), compared to $13 / 42$ (31\%) recorded with renal criteria ANA IIF alone. ANA IIF +, 158/254 (62\%) biopsy proven nephritis (LN), rather than relying on proteinuria for diagnosis, compared to $5 / 13(38 \%)$ of ANA IIF alone biopsy proven LN. Remaining 8 ANA IIF alone, uPCR exceeded $0.5 \mathrm{~g}$ in 1 of $44(2 \%)$ encounters. Low incidence of proteinuria explained by complete renal response or prior proteinuria misconstrued as evidence of LN. In comparison, uPCR $>0.5 \mathrm{~g}$ was present in 694 of 1157 (60\%) encounters in ANA IIF +, casting doubt on validity of LN diagnosis in 8 ANA IIF alone without biopsy.
Leucopenia, lymphocytopenia, AITP, AIHA statistically less ANA IIF alone compared to ANA IIF + ; $24 \%$ vs $36 \%$, $29 \%$ vs $40 \%, 7 \%$ vs $15 \%$ and $0 \%$ vs $7 \%$, respectively. 42 patients with ANA IIF alone prevalence of potentially misattributed (e.g. not result of IMID) clinical criteria such as photosensitivity (64\%) and malar rash (60\%) greater compared to ANA IIF +, 38\% and 44\%, respectively. Prevalence oral ulcers, DLE, arthritis, serositis, seizures and psychosis equivalent in both.

\begin{tabular}{|c|c|c|c|}
\hline & ANA IIF + & $\begin{array}{l}\text { ANA IIF } \\
\text { alone }\end{array}$ & $P$ Value \\
\hline $\mathrm{n}$ & $548 / 590(93 \%)$ & $42 / 590(7 \%)$ & $<0.001$ \\
\hline Nephritis & $254 / 548(46 \%)$ & $13 / 42(31 \%)$ & $=0.0442$ \\
\hline GN by biopsy & 158/254 (62\%) & $5 / 13(38 \%)$ & $=0.1222$ \\
\hline GN by proteinuria & 96/254 (38\%) & $8 / 13(62 \%)$ & $=0.1222$ \\
\hline Visits with & $694 / 1157$ & $1 / 44(2 \%)$ & $<0.0001$ \\
\hline $\mathrm{uPCR}>0.5$ & $(60 \%)$ & & \\
\hline Malar rash & $44 \%$ & $60 \%$ & $=0.1163$ \\
\hline Photosensitivity & $38 \%$ & $64 \%$ & $=0.0018$ \\
\hline AITP & $15 \%$ & $7 \%$ & $=0.0544$ \\
\hline AlHA & $7 \%$ & $0 \%$ & $<0.0001$ \\
\hline Leucopenia & $36 \%$ & $24 \%$ & $=0.0254$ \\
\hline Lymphocytopenia & $40 \%$ & $29 \%$ & $=0.0888$ \\
\hline Oral ulcers & $24 \%$ & $26 \%$ & NS \\
\hline DLE & $22 \%$ & $33 \%$ & NS \\
\hline Arthritis & $70 \%$ & $69 \%$ & NS \\
\hline Serositis & $27 \%$ & $31 \%$ & NS \\
\hline Seizures & $4 \%$ & $2 \%$ & NS \\
\hline Psychosis & $2 \%$ & $5 \%$ & NS \\
\hline
\end{tabular}

ACR Clinical Criteria ANA IIF alone vs ANA IIF + 
Conclusions ANA IIF alone rare and patients infrequently develop nephritis, leucopenia, lymphocytopenia, AITP, AIHA. In patients ANA IIF alone attribution of ACR/SLICC clinical criteria needs to be point of emphasis and unless biopsy proven, alternative explanation for proteinuria should be considered. Data argues inclusion criteria for clinical trials, rather than allowing ANA IIF alone or dsDNA, may need to require ANA IIF and at least one of the following (dsDNA, Sm, Ro, $\mathrm{La}$, aPL, or hypocomplementemia) to avoid enrolling patients that do not have SLE.

Funding Source(s): None

\section{INCREASED WORK LOSS DURING PREGNANCY IN WOMEN WITH SYSTEMIC LUPUS ERYTHEMATOSUS COMPARED TO MATCHED HEALTHY CONTROLS}

${ }^{1}$ Birgit S Blomjous, ${ }^{2}$ Marieke M ter Wee, ${ }^{1}$ Alexandre E Voskuyl, ${ }^{3}$ Johanna IP de Vries, ${ }^{1}$ Irene EM Bultink*. ${ }^{1}$ Department of Rheumatology, Amsterdam Rheumatology and immunology Center, Amsterdam UMC, location VUmc, Amsterdam, Netherlands; 'Department of Epidemiology and Biostatistics, Amsterdam UMC, location VUmc, Amsterdam, Netherlands; ${ }^{3}$ Department of Obstetrics and Gynecology, Amsterdam UMC, location VUmc, Amsterdam, Netherlands

10.1136/lupus-2019-Ism.65

Background Women with systemic lupus erythematosus (SLE) might be more vulnerable to reduce or stop working during pregnancy because of the increased risk of pregnancy complications compared to the general population. However, no data on work loss during pregnancy and return to work after maternity leave in patients with SLE are available. We aimed to investigate several work outcomes during and after pregnancy in women with SLE compared to matched pregnant controls.

Methods A case-control study on employment was performed in pregnant women with SLE and matched controls. Matching criteria were age, year of delivery, and number of living infants. Employment was defined as having 8 hours/week of paid work before conception. Four work outcomes were investigated: interruption of work for $>1$ week during pregnancy, complete cessation of work during pregnancy for $>1$ week until delivery, reduction in working hours during pregnancy, and the time in weeks to return to work after maternity leave.

Results A total of 42 women were included (21 SLE patients, 21 controls). Mean SELENA-SLEDAI before pregnancy in SLE patients was 2.6 (SD 2.3). Interruption of work for $>1$ week or completely stop working during pregnancy occurred in 10 women with SLE compared to 2 controls $(\mathrm{OR}=8.6,95 \% \mathrm{CI}[1.6-46.8], \mathrm{p}=0.012)$. From the women who completely stopped working until delivery $(n=8), 7$ women had SLE versus 1 control $(\mathrm{OR}=1.4$, 95\% CI [0.0728.1], $\mathrm{p}=0.826$ ). In addition, in women continuing work, reduction of working hours during pregnancy occurred in 5 women with SLE versus 3 controls $(\mathrm{OR}=1.9$, 95\% CI [0.49.1], $\mathrm{p}=0.436$ ).

After delivery, the median (IQR) duration of return to work after maternity leave was $4(0-6.8)$ weeks after maternity leave for women with SLE and 2 (0-4) weeks later for controls (Mann-Whitney $U$ test, $p=0.977$ ). No difference in number of women with delay of return to work after maternity leave (yes/no) was found between women with SLE and controls ( $n=9$ versus $n=11$, respectively, OR $=1.0,95 \% C I$ [0.3-3.7], $\mathrm{p}=0.973)$.
Conclusions Pregnant women with SLE more frequently completely stop working or reduce working hours compared to matched healthy controls. These findings warrant improved counseling of these women and attention of health care providers, including company doctors.

Funding Source(s): None

\section{NEUROLOGIC MANIFESTATIONS OF THE ANTIPHOSPHOLIPID SYNDROME AND RESPONSE TO HYDROXYCHLOROQUINE: A DESCRIPTIVE STUDY}

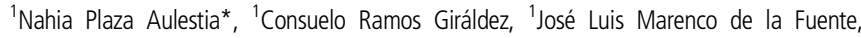
${ }^{2}$ Mercedes Romera Tellado. ${ }^{1}$ Valme Hospital (Rheumatology unit); ${ }^{2}$ Valme Hospital (Neurology unit)

\subsection{6/lupus-2019-Ism.66}

Background The primary antiphospholipid syndrome (APS) is characterized by arterial and/or venous thrombosis and pregnancy morbidity in the presence of anticardiolipin antibodies $(\mathrm{aCL})$ and/or lupus anticoagulant (LA). In addition to prothrombotic effects of aPL on the brain, there are immunologic effects with proof of direct binding of aPL to various types of brain cells, presented as cognitive dysfunction (CD), migraine, seizure, multiple sclerosis-like syndrome (MS-like), transverse myelitis (TM), movement disorders, or psychiatric symptoms.

Methods We examined 3 patients who were diagnosed primary APS.The patients were assessed with careful history taking, physical examination, blood laboratory evaluation, and MRI or head-CT.Our main objective was to describe neurologic manifestations of APS in our patients at Valme University Hospital and the response to treatment with hydroxychloroquine

Results Among the 3 patients, there was male preponderance with 3 men and no-women.The mean age of presentation was $33.66 \pm 5.2$ years (range, 2350 years) and with a current mean age of $37 \pm 14,93$ years. There was no mortality in our series. One of them debuted with sudden loss of consciousness along with jaw stiffness and posterior amnesia.The other one presented prickling sensation and involuntary movement of his right upper limb extending to right lower limbs without posterior generalization, and the third patient consulted with frontal, pulsating headache and binocular diplopia.100\% were LA positive with prolongated Activated Partial Thromboplastin Time and dilute Russell viper venom time and negative for ANA with no collagenosis sign or symptoms. Neither presented complement alteration or cerebrospinal fluid variation. We observed positivity for aCL in one patient (33\%). In 2 of 3 patients (66.66\%), cortico-subcortical space occupant lesions (SOL) were observed, some of them with contrast enhancement, mimicking demyelinating lesion, while the other patient didn't present any abnormality in the MRI images.All patients presented an appropriate response to treatment with prednisone, in downward treatment regimen, aspirin and hydroxychloroquine $200 \mathrm{mg}$ twice a day. At the four-year follow-up, all of them remain asymptomatic. We observed a lessening of SOL in MRI images due to the treatment in two patients but the third one presented new lesions due to suspension od prednisone, which was reintroduced, lessening of SOL.

Conclusions The neurological affection presented in APS can mimic multiple sclerosis symptoms and it is difficult to differentiate both entities That is why aPL determination should be part of screening tests and should not be delayed if the 